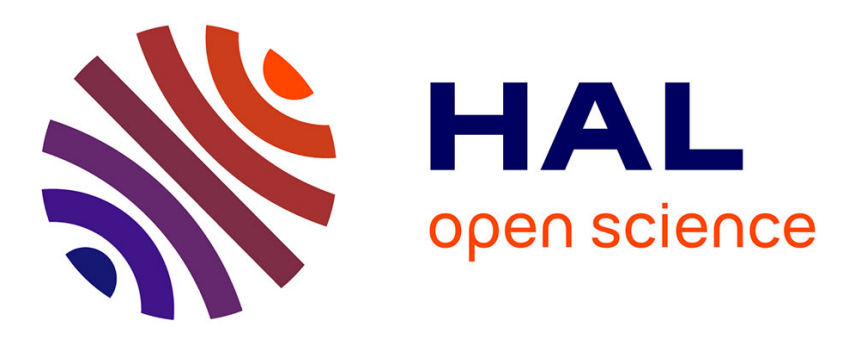

\title{
Solar Wind Charge Exchange Emission from the Helium Focusing Cone: Model to Data Comparison
}

\author{
Dimitra Koutroumpa, M.R. Collier, K.D. Kuntz, Rosine Lallement, S.L.
}

Snowden

\section{- To cite this version:}

Dimitra Koutroumpa, M.R. Collier, K.D. Kuntz, Rosine Lallement, S.L. Snowden. Solar Wind Charge Exchange Emission from the Helium Focusing Cone: Model to Data Comparison. The Astrophysical Journal, 2009, 697 (2), pp.1214-1225. 10.1088/0004-637X/697/2/1214 . hal-00399623

\section{HAL Id: hal-00399623 https://hal.science/hal-00399623}

Submitted on 7 Jul 2020

HAL is a multi-disciplinary open access archive for the deposit and dissemination of scientific research documents, whether they are published or not. The documents may come from teaching and research institutions in France or abroad, or from public or private research centers.
L'archive ouverte pluridisciplinaire HAL, est destinée au dépôt et à la diffusion de documents scientifiques de niveau recherche, publiés ou non, émanant des établissements d'enseignement et de recherche français ou étrangers, des laboratoires publics ou privés. 


\title{
SOLAR WIND CHARGE EXCHANGE EMISSION FROM THE HELIUM FOCUSING CONE: MODEL TO DATA COMPARISON
}

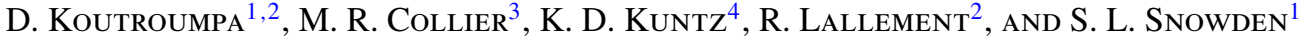 \\ ${ }^{1}$ Code 662, NASA/Goddard Space Flight Center, Greenbelt, MD 20771, USA \\ 2 Service d'Aéronomie, UMR 7620, CNRS, Université de Versailles Saint-Quentin, BP3, 91371 Verrières-le-Buisson, France \\ ${ }^{3}$ Code 673, NASA/Goddard Space Flight Center, Greenbelt, MD 20771, USA \\ ${ }^{4}$ Henry A. Rowland Department of Physics and Astronomy, The Johns Hopkins University, 366 Bloomberg Center, 3400 N. Charles Street, Baltimore, \\ MD 21218, USA \\ Received 2008 December 19; accepted 2009 March 13; published 2009 May 11
}

\begin{abstract}
A model for heliospheric solar wind charge exchange (SWCX) X-ray emission is applied to a series of XMM-Newton observations of the interplanetary focusing cone of interstellar helium. The X-ray data are from three coupled observations of the South Ecliptic Pole (SEP; to observe the cone) and the Hubble Deep FieldNorth (HDF-N, to monitor global variations of the SWCX emission due to variations in the solar wind (SW)) from the period 2003 November 24 to December 15. There is good qualitative agreement between the model predictions and the data, after the SEP data are corrected using the HDF-N data, with the maximum SWCX flux observed at an ecliptic longitude of $\sim 72^{\circ}$, consistent with the central longitude of the He cone. We observe a total excess of $2.1 \pm 1.3$ line unit (LU) in the OVII line and $2.0 \pm 0.9$ LU in the O VIII line. However, the SWCX emission model, which was adjusted for SW conditions appropriate for late 2003, predicts an excess from the He cone of only $0.5 \mathrm{LU}$ and $0.2 \mathrm{LU}$, respectively, in the O VII and O VIII lines. We discuss the model to data comparison and provide possible explanations for the discrepancies. We also qualitatively re-examine our SWCX model predictions in the $\frac{1}{4} \mathrm{keV}$ band with data from the ROSAT All-Sky Survey toward the North Ecliptic Pole and SEP, when the He cone was probably first detected in soft X-rays.
\end{abstract}

Key words: solar wind - X-rays: general

Online-only material: color figure

\section{INTRODUCTION}

Solar wind charge exchange (SWCX) X-ray emission in the heliosphere originates when a high charge state ion of the solar wind (SW) interacts with a neutral atom and gains an electron in a highly excited state which then decays by emission of an X-ray with a characteristic energy of the ion. SWCX emission occurs over a wide range of spatial scales including comets (where such emission was first conclusively observed, e.g., Lisse et al. 1996), the near-Earth environment where the neutrals are exospheric material in and near the magnetosheath (e.g., Cravens et al. 2001; Snowden et al. 2009), and throughout the solar system where the target atoms are from the interstellar medium (ISM; e.g., Cox 1998; Cravens 2000; Smith et al. 2005). SWCX emission comprises a significant part of the diffuse X-ray background observed at energies $\lesssim 1 \mathrm{keV}$, and can be either a signal of interest in studies of the solar system or an unfortunate contamination component in studies of the hot, diffuse plasmas in the Milky Way and beyond.

In theory, SWCX emission exhibits very distinct spectral characteristics with respect to thermal plasma emission. For instance, the line intensity ratios in triplets of the He-like ions $\mathrm{O}$ VII and Ne IX are very different and could be used as spectral diagnostics to separate the two mechanisms (e.g., Lallement 2009, and references therein). Future missions which include nondispersive microcalorimeter spectrometers will be able to separate thermal from SWCX emission using the triplets (e.g., Snowden 2009). However for diffuse sources, with the spectral resolution of current missions it is impossible, in general, to distinguish individual SWCX X-rays from those with a more distant origin, and alternative diagnostic information must be used to separate the components of the X-ray background. Temporal variation can be used to a certain extent depending on the length of an observation, and indeed this method was used in cleaning the ROSAT All-Sky Survey (RASS; Snowden et al. 1997). SWCX emission from the near-Earth environment will track relatively well variations in the SW flux as measured by the Advanced Composition Explorer (ACE; Stone et al. 1998) and WIND (Ogilvie et al. 1995) instruments. However, identifying and modeling a temporally variable component can only address a fraction of the SWCX emission. Emission from interactions with interstellar (IS) neutrals drifting through the solar system will typically have minimal temporal variation as the observed flux along any line of sight (LOS) is an integral over weeks of SW conditions. Because of the long (relative to a typical X-ray observation) timescales of any expected variation, heliospheric SWCX effectively contributes a zero-level offset to observations of the diffuse X-ray background of more distant origin.

While there are models for SWCX emission from the heliosphere, testing them is problematic. Koutroumpa et al. (2007), using a self-consistent model of the heliospheric SWCX emission, managed to associate observed discrepancies in XMMNewton and Suzaku observations separated by several years with solar cycle-scale variations. A recent paper (Snowden et al. 2009) was relatively successful at using an XMM-Newton observation to test a model (Robertson \& Cravens 2003) for emission from the near-Earth environment. In this paper, we use a different series of XMM-Newton observations to search for SWCX emission from the solar system. Specifically, we use multiple observations of the South Ecliptic Pole (SEP) as Earth passes over the ISM helium focusing cone downstream of the Sun to 
Table 1

XMM-Newton Observation Details and Fitted Line Fluxes in LU

\begin{tabular}{|c|c|c|c|c|c|c|c|c|c|}
\hline ObsId & Observation Start & $\lambda_{\mathrm{Obs}}{ }^{\mathrm{a}}$ & $\alpha$ & $\delta$ & Exposure (ks) & Good Time (ks) & $\begin{array}{c}\text { Fitted } \\
\text { O viI Flux }\end{array}$ & $\begin{array}{c}\text { Fitted } \\
\text { O viII Flux }\end{array}$ & Field \\
\hline 0162160101 & 2003 Nov 24 20:07:55 & 61 & 060009.36 & -663415.7 & 13.44 & 11.90 & $8.3 \pm 0.5$ & $3.8 \pm 0.2$ & SEP \\
\hline 0162160201 & 2003 Nov 24 09:18:35 & 61 & 123701.02 & 621257.1 & 15.25 & 12.89 & $8.9 \pm 0.6$ & $4.3 \pm 0.4$ & HDF-N \\
\hline 0162160301 & 2003 Dec 5 20:47:15 & 72 & 060007.63 & -663426.8 & 11.45 & 8.57 & $9.7 \pm 0.7$ & $4.1 \pm 0.4$ & SEP \\
\hline 0162160401 & 2003 Dec 6 12:38:23 & 72 & 123701.02 & 621306.2 & 10.85 & 9.62 & $8.0 \pm 0.6$ & $2.1 \pm 0.3$ & HDF-N \\
\hline 0162160501 & 2003 Dec 14 14:26:09 & 82 & 060006.48 & -663434.8 & 11.45 & 9.32 & $8.6 \pm 0.7$ & $3.5 \pm 0.4$ & SEP \\
\hline 0162160601 & 2003 Dec 14 23:15:09 & 82 & 123701.70 & 621316.0 & 43.17 & 10.06 & $7.0 \pm 0.5$ & $1.8 \pm 0.3$ & HDF-N \\
\hline 0111550201 & 2001 May 18 22:17:34 & 238 & $12: 36: 50.00$ & $62: 13: 12.0$ & 41.88 & 34.89 & $10.4 \pm 0.5$ & $2.6 \pm 0.2$ & HDF-N \\
\hline 0111550401 & 2001 Jun 1 08:16:36 & 251 & $12: 36: 57.00$ & $62: 13: 30.0$ & 91.99 & $26.73^{b}$ & $8.9 \pm 0.5$ & $2.8 \pm 0.2$ & HDF-N \\
\hline
\end{tabular}

Notes.

a Observer ecliptic longitude.

b Data from the low SWCX emission part of the observation.

search for a correlation with the expected variation from the model.

\section{OBSERVATIONS}

\subsection{Helium Focusing Cone}

The flow of IS neutrals through the solar system is due to the motion of the heliosphere at about $25 \mathrm{~km} \mathrm{~s}^{-1}$ through the local IS cloud. This material, a gas of mostly hydrogen atoms with about $15 \%$ helium, flows from the direction of $\lambda$, $\beta \sim 252^{\circ}, 9^{\circ}\left(\alpha, \delta \sim 252^{\circ},-14^{\circ}\right.$; Lallement et al. 2005). This places the Earth upstream of the Sun in the IS neutral flow in early June and downstream in early December every year (Gruntman 1994). Although both radiation pressure and gravity affect the hydrogen trajectories (e.g., Quémerais et al. 1999; Lallement 1999), only gravity significantly affects the helium trajectories which execute Keplerian orbits and form a "focusing cone" downstream of the Sun, resulting in a localized downstream enhancement of helium observed annually by Earth orbiting and L1 spacecraft (Bzowski et al. 1996; Frisch 2000).

Many diverse sets of observations have confirmed this helium focusing cone phenomenon, and have put strict constraints on the helium flow parameters (density, temperature, velocity vector), including direct neutral gas measurements (Witte 2004) and UV backscattering (Lallement et al. 2004; Vallerga et al. 2004) as well as pickup ions (Gloeckler et al. 2004) which form when the neutral helium becomes ionized primarily from solar photons and subsequently "picked up" by the SW flow (Moebius et al. 1985). Of these, pickup ions have been particularly useful because they display a clear spectral signature, a cutoff at two times the SW speed resulting from their low initial speed $\left(\sim 25 \mathrm{~km} \mathrm{~s}^{-1}\right)$ in comparison to the SW speed $\left(\sim 400 \mathrm{~km} \mathrm{~s}^{-1}\right)$. However, their low count rates also necessitate aggressive averaging, commonly 30 day running averages, to smooth out fluctuations.

Recently, Collier et al. (2004), based on an analysis of several diverse data sets, proposed the presence of a secondary stream of neutral atoms at $1 \mathrm{AU}$ located at a higher ecliptic longitude by $10^{\circ}-40^{\circ}$ than the primary stream described above (see also Wurz et al. 2004). These neutrals seem to be far more energetic than the nominal He flow. Very recent observations from the pair of STEREO spacecraft (Wang et al. 2008) showing two peaks in the 4-20 keV neutral hydrogen atom flux, one primary peak at $245^{\circ}$ close to the nominal upstream direction and another secondary peak shifted $16^{\circ}$ toward higher ecliptic longitudes than the nominal upstream direction, appear to confirm this prediction.
Additionally, Mars Express has observed a neutral atom signal consistent with this secondary stream proposal (Holmström et al. 2008). Nevertheless, all observations of slow neutrals show only one cone, and the secondary cone, should it exist, must consist of high-velocity neutrals which are not well focused.

Because high charge state SW ions will charge exchange with the enhanced helium in the downstream region emitting soft X-rays, the helium focusing cone should be observable in soft X-rays (Cravens et al. 2001). Indeed, the RASS data (Snowden et al. 1995) when observing within $2^{\circ}$ of the SEP show enhanced emission in early December (see Section 2.4) with some evidence for a secondary peak about 10 days later (or $10^{\circ}$ higher in ecliptic longitude) than the expected location of the helium focusing cone (see Figure 6). These ROSAT data in part motivated the XMM-Newton observations of the helium focusing cone and particularly the day of year of the third observation.

\subsection{X-ray Data}

Three pairs of XMM-Newton calibration observations, matched pointings of the SEP (to observe the focusing cone) and of the Hubble Deep Field-North (HDF-N), were approved by the project and scheduled for late 2003 (see Table 1 for the observation details). The coupled observations of the HDF-N were included to serve as controls to monitor the SWCX emission variation not associated with the focusing cone (e.g., due to variations in the SW flux and composition) which could arise from Earth's magnetosheath or more generally from the heliosphere. In Figure 1, we show the geometry differences between the XMM-Newton SEP $\left(\beta=-90^{\circ}\right)$ and HDF-N $(\lambda, \beta=148$, $57^{\circ}$ ) observations, associated with the He cone structure.

The orbit of XMM-Newton is highly elongated (perigee $\sim 10^{4} \mathrm{~km}$, apogee $\sim 10^{5} \mathrm{~km}$ ) and inclined (inclination $\sim-40^{\circ}$ ) relative to the equatorial plane. In late November and early December, the time period when Earth is closest to the focusing cone and therefore the time for these observations, the apogee lies in the antisolar direction. This is fortuitous for two reasons: since observations take place away from perigee any observed SWCX emission from the magnetosheath (well down the flanks of the magnetosheath and away from the subsolar point) is significantly reduced (e.g., Robertson \& Cravens 2003) and the likelihood of soft proton contamination is also reduced (Kuntz $\&$ Snowden 2008).

We reduced the EPIC data using the XMM-Newton ESAS analysis package developed by S. L. Snowden and K. D. Kuntz ${ }^{5}$

\footnotetext{
5 http://heasarc.gsfc.nasa.gov/docs/xmm/xmmhp_xmmesas.html
} 

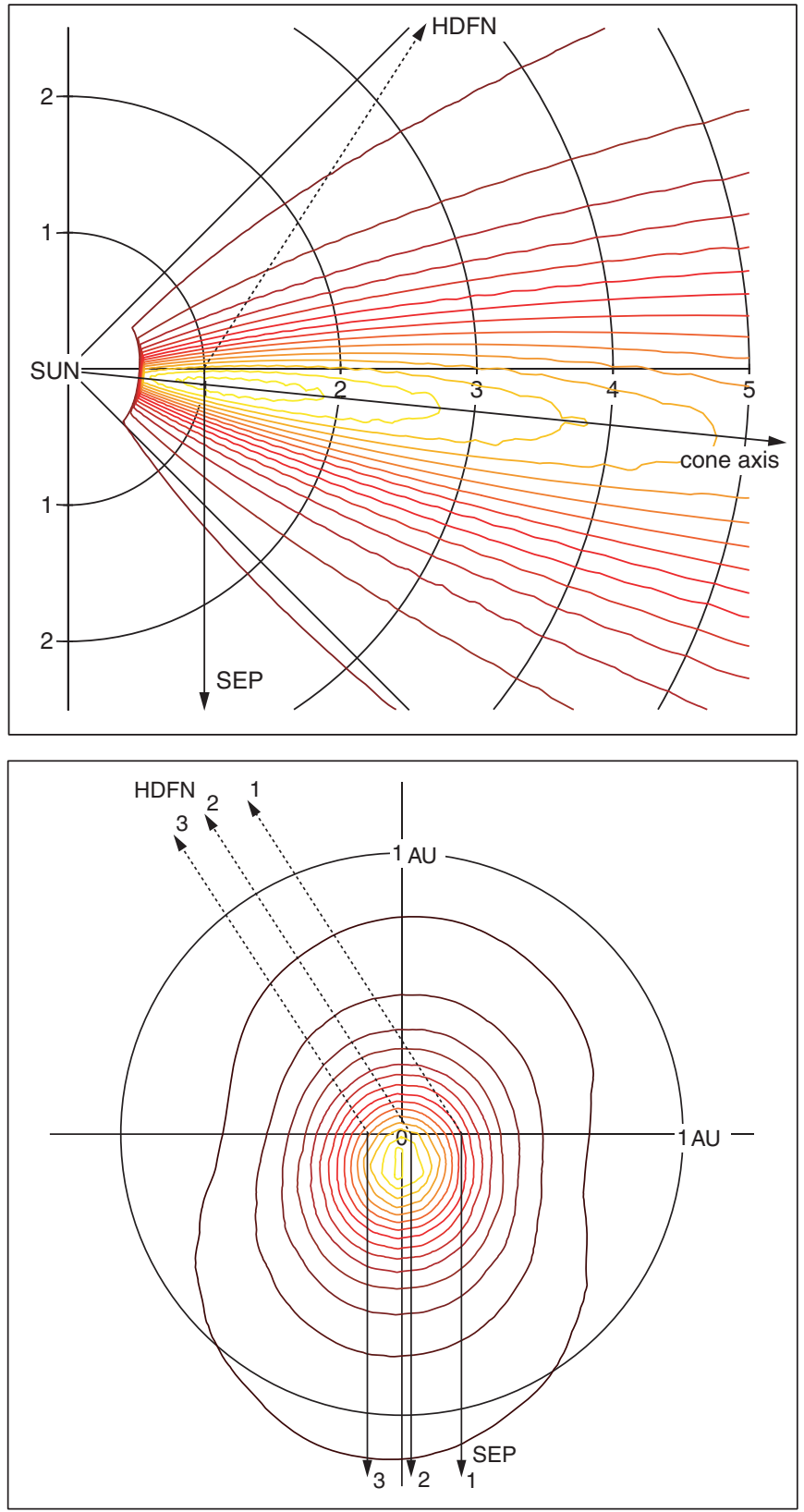

Figure 1. Upper panel: XMM-Newton SEP and HDF-N observation geometry difference as seen perpendicular to the Sun-Earth direction, in a cut through the He cone central longitude $\left(\sim 74^{\circ}\right)$. Lower panel: same as the upper panel except the vantage point is on the Sun looking through $\lambda \sim 74^{\circ}$, and all three observation groups are shown as the Earth (observer) crosses the He cone on its orbit (at $\sim 1$ AU from the Sun). The isodensity contours in both panels start at $0.073 \mathrm{~cm}^{-3}$ and decrease to $0.017 \mathrm{~cm}^{-3}$ in $0.003 \mathrm{~cm}^{-3}$ steps.

(A color version of this figure is available in the online journal.)

as demonstrated in Snowden et al. (2008). The data were first screened for variations in the light curve which removed excesses in the normal internal particle background and more commonly contamination by soft protons (Kuntz \& Snowden 2008). Next, data from the full field of view were extracted after the exclusion of point sources to a limit to $10^{-14} \mathrm{erg} \mathrm{cm}^{-2} \mathrm{~s}^{-1}$. Finally, model particle background spectra were produced for subtraction during the spectral fitting process.

After extraction the spectra were then fitted using a model which included two thermal components (an unabsorbed $\sim 0.1 \mathrm{keV}$ model for local emission and an absorbed $\sim 0.3 \mathrm{keV}$

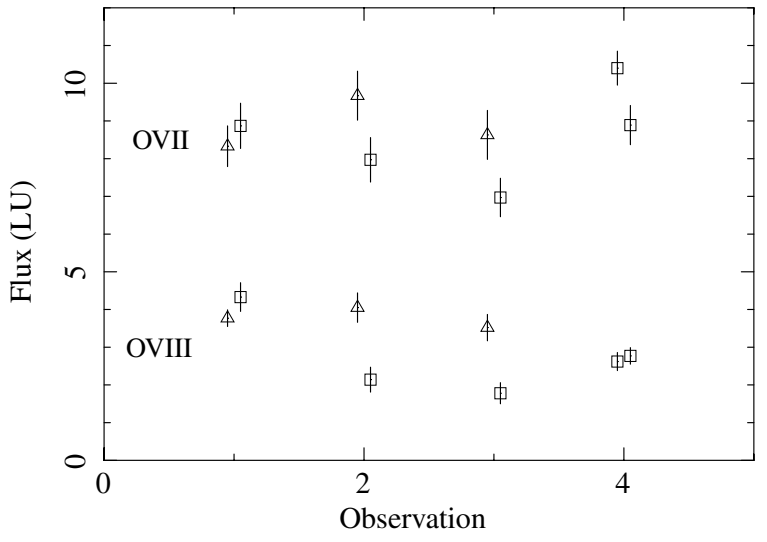

Figure 2. Fitted values for the O VII (upper grouping of points) and $\mathrm{O}$ VIII line fluxes (lower grouping of points). Data points with a triangular symbol are in the direction of the SEP and data points with a rectangular symbol are in the direction of the HDF-N. The pairs of points at observations 1-3 are ObsIds (0162160101, 0162160201), (0162160301, 0162160401), and (0162160501, $0162160601)$, respectively. The observation 4 data points are from two HDF-N spectra $(0111550201,0111550401)$ from Snowden et al. (2004).

model for the more distant disk and halo emission), an absorbed power law representing the cosmic background, monochromatic lines at $1.49 \mathrm{keV}$ and $1.75 \mathrm{keV}$ representing the internal fluorescent $\mathrm{Al} \mathrm{K} \alpha$ and $\mathrm{Si} \mathrm{K} \alpha$ background, and a power law not folded through the instrumental response representing any residual soft proton contamination left after the screening process. Xspec APEC thermal spectral models with variable abundances were used where the abundance of oxygen was set to zero and the other abundances were fixed at 1 (based on the assumption that oxygen will dominate any SWCX emission in the XMM-Newton bandpass). Monochromatic lines at $0.57 \mathrm{keV}$ and $0.65 \mathrm{keV}$ were added to the model to represent the observed oxygen emission from both SWCX and more distant cosmic emission, the latter which produces a constant contribution. The absorption of the halo thermal components and the power law were fixed at the Galactic values. Two other monochromatic lines were also added, $\mathrm{C}$ VI at $0.46 \mathrm{keV}$ and another $\mathrm{O}$ VIII line at $0.81 \mathrm{keV}$. The addition of the $\mathrm{C}$ VI line marginally improved the fit but left the $\chi_{v}^{2}$ value unchanged with detections at the $<2 \sigma$ level. The $\mathrm{O}$ vIII line at $0.81 \mathrm{keV}$ was insignificant. A spectrum derived from the RASS data was simultaneously fitted with the oxygen emission coming from the thermal models rather than the monochromatic lines. (The RASS data primarily constrain the low-temperature thermal component.)

All of the SEP data and all of the HDF-N data were fitted simultaneously allowing only the SWCX oxygen Gaussians, instrumental $\mathrm{Al}$ and $\mathrm{Si}$ Gaussians, and soft proton powerlaw components to vary between observations. The model components representing the cosmic background were assumed to be constant. Table 1 also lists the fitted values for the $\mathrm{O}$ vII and $\mathrm{O}$ viII flux in line units (LUs, photons $\mathrm{cm}^{-2} \mathrm{~s}^{-1} \mathrm{sr}^{-1}$ ). Also included are results from the spectral fits from two of the HDFN spectra from Snowden et al. (2004). The data are also plotted in Figure 2.

The variation in the $\mathrm{O}$ VII and $\mathrm{O}$ VIII line strengths (and their ratios) among the different HDF-N observations demonstrates the difficulty of determining the line strengths for diffuse Galactic emission using single observations. Although the SW proton fluxes and ion ratios are similar for ObsIds 0162160201 and 0111550201 (the first and next to last HDF-N points), the $0111550201 \mathrm{O}$ VII flux is higher, perhaps due to a greater 


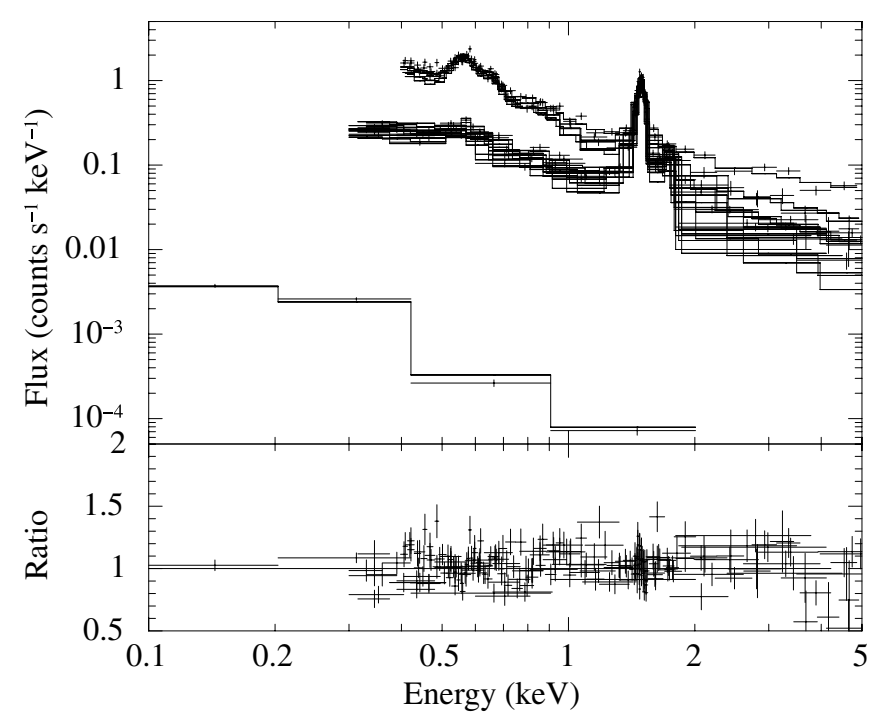

Figure 3. Spectral fit for the SEP spectra. The upper set of data and curves are the three PN spectra, the middle set of data and curves are the six MOS1 and MOS 2 spectra, and the bottom is the RASS spectrum.

Table 2

Normalized Line Fluxes in LU

\begin{tabular}{lcccc}
\hline \hline ObsId & $\begin{array}{c}\text { Normalized }^{\mathrm{a}} \\
\text { O viI Flux }\end{array}$ & $\begin{array}{c}\text { Normalized }^{\mathrm{a}} \\
\text { O viII Flux }\end{array}$ & $\begin{array}{c}\text { Normalized }^{\mathrm{b}} \\
\text { O viI Flux }\end{array}$ & $\begin{array}{c}\text { Normalized }^{\mathrm{b}} \\
\text { O viII Flux }\end{array}$ \\
\hline 0162160101 & $6.5 \pm 0.8$ & $1.6 \pm 0.3$ & $7.2 \pm 0.8$ & $1.7 \pm 0.3$ \\
0162160301 & $8.5 \pm 1.0$ & $3.4 \pm 0.8$ & $9.3 \pm 1.0$ & $3.7 \pm 0.8$ \\
0162160501 & $8.6 \pm 0.7$ & $3.5 \pm 0.4$ & $8.6 \pm 0.7$ & $3.5 \pm 0.4$ \\
\hline
\end{tabular}

Notes.

a Scaled only by the observed variation of the HDF-N flux.

b Scaled by the observed variation of the HDF-N flux and then scaled again by the model variation of the HDF-N SWCX flux.

magnetosheath SWCX contribution. The O VIII shows the opposite trend, but since the $\mathrm{O}^{8+}$ population for both observations was below the $A C E$ sensitivity, we have no means of determining the cause of this difference.

Figure 3 shows the spectral fits of the SEP data (the fits to the HDF-N data were similar). The fits are reasonably good for the cosmic background with a $\chi_{v}^{2}$ value of 1.24 for 902 degrees of freedom ( $\chi_{v}^{2}$ value of 1.25 for 771 degrees of freedom for the HDF-N data). Of note in the plot are the significantly better statistics of the PN data with the O VII line clearly seen at $E \sim 0.57 \mathrm{keV}$ and with the $\mathrm{O}$ VIII line forming the $E \sim 0.65 \mathrm{keV}$ hump on the shoulder of the O VII line.

The fitted fluxes in Figure 2 show significant scatter. One would expect the second SEP observation (on-cone) to be higher than both the first (off-cone) and the third (the position of the secondary cone proposed by Collier et al. 2004). The expected trend is only marginally detected due to the large uncertainties. Due to the observing geometry (see Figure 1), the HDF-N measurements should be almost completely unaffected by the helium focusing cone, and should be roughly constant save for the variations in the SW flux. We therefore used the HDF-N data to normalize the SEP data. As noted above, the observations took place while the XMM-Newton satellite was on the opposite side of Earth from the Sun minimizing the SWCX emission from in and near the magnetosheath (Robertson \& Cravens 2003) allowing the observed SWCX X-ray emission to be dominated by the heliosphere.

Given this assumption we divided each SEP measurement by the corresponding HDF-N value, and then normalized to the

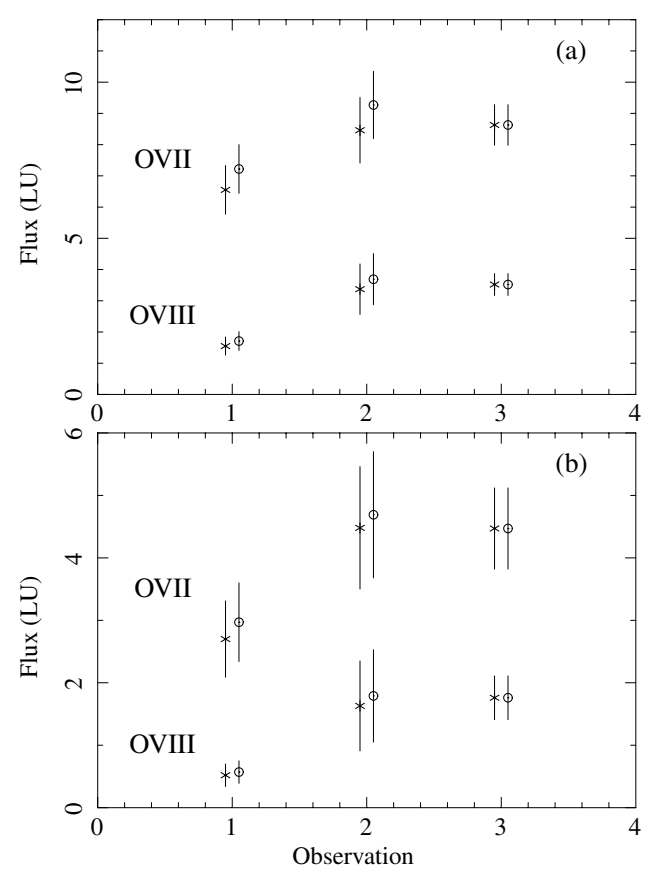

Figure 4. (a) SEP line fluxes after using the HDF-N data to normalize flux for the O VII (upper grouping of points) and O VIII line fluxes (lower grouping of points). The data are from Table 2. Data points with an " $\mathrm{X}$ " symbol show the results when the HDF-N SWCX flux is assumed to be constant. The points with a circle symbol include a further scaling to account for the model variation in the direction of the HDF-N, see the text for the details. (b) Same as (a) except for the assumption that half of the minimum observed flux in both directions (data from Table 3) has an origin outside of the heliosphere. This distant emission has been subtracted from the data.

value of the third SEP observation (the observation with the lowest $\mathrm{O}$ VII and $\mathrm{O}$ VIII fluxes). The results are listed in Table 2 and are shown by the X symbols in Figure 4(a). However, our model prediction for the HDF-N SWCX flux does vary by about 10\% (see Table 5 and Figure 7) with the intensity being lowest for the last observation. We use these model HDF-N fluxes to add a second normalization to the SEP fluxes where the results are also listed in Table 2 and shown by the circles in Figure 4(a). The total on-cone SWCX excess (with respect to the off-cone directions) is $2.1 \pm 1.3 \mathrm{LU}$ for $\mathrm{O}$ VII and $2.0 \pm 0.9 \mathrm{LU}$ for $\mathrm{O}$ vIII. There are a couple of caveats in using this approach. First, we assume that the heliospheric SWCX oxygen line emission dominates the observed flux. A significant contribution either from the Milky Way or yet more distant emission or a constant flux from the heliopause would decrease the accuracy of the scaling by adding a constant offset. Second, we assume that the $\mathrm{SW}$, both flux and abundances, on average is the same for both the northern and southern solar hemispheres.

Since the observed fields (SEP and HDF-N) do not point at any significantly dense IS neutral clouds, the diffuse X-ray emission detected is not "shadowed," and should include emission from the more distant galactic halo and extragalactic sources. To gauge the effect of possible more distant emission, we recalculated the normalized line intensities with the assumption that half of the minimum observed fluxes in both directions and both spectral lines originates beyond the heliosphere. The results are listed in Table 3 and plotted in Figure 4(b). Not surprisingly the sense of the variation is the same as before and the only significant difference is that the fluxes are reduced by a factor of 2 . In this case, the total on-cone SWCX excess is found to be $1.7 \pm$ 1.2 LU and $1.2 \pm 0.7 \mathrm{LU}$ for O VII and O VIII, respectively. 


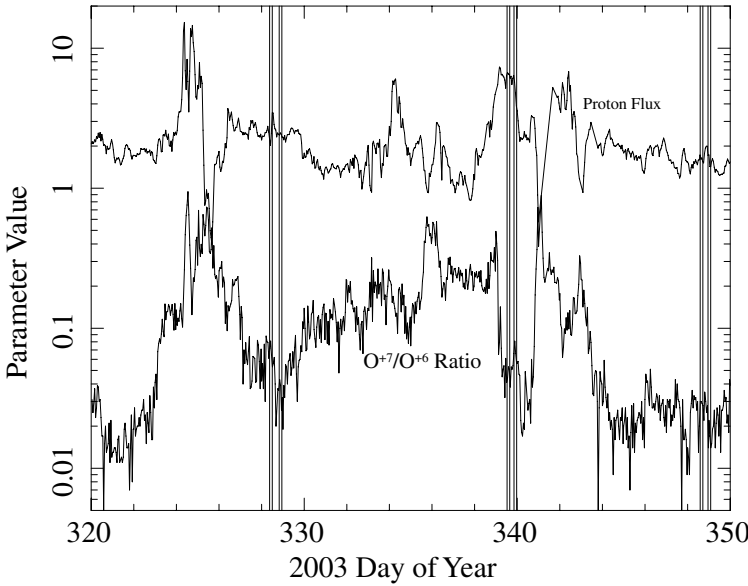

Figure 5. ACE SW proton flux (upper curve, units of $10^{8}$ particles $\mathrm{cm}^{-2} \mathrm{~s}^{-1}$ ) and the $\mathrm{O}^{7+} / \mathrm{O}^{6+}$ density ratio data (lower curve) covering the time interval of the observations. The vertical lines show the individual observation intervals.

Table 3

Offset Subtracted and Normalized ${ }^{\mathrm{a}}$ Line Fluxes in LU

\begin{tabular}{lcccc}
\hline \hline ObsId & $\begin{array}{c}\text { Normalized }^{\mathrm{b}} \\
\text { O vII Flux }\end{array}$ & $\begin{array}{c}\text { Normalized }^{\mathrm{b}} \\
\text { O vIII Flux }\end{array}$ & $\begin{array}{c}\text { Normalized }^{\mathrm{c}} \\
\text { O vII Flux }\end{array}$ & $\begin{array}{c}\text { Normalized }^{\mathrm{c}} \\
\text { O vIII Flux }\end{array}$ \\
\hline 0162160101 & $2.7 \pm 0.6$ & $0.5 \pm 0.2$ & $3.0 \pm 0.6$ & $0.6 \pm 0.2$ \\
0162160301 & $4.5 \pm 1.0$ & $1.6 \pm 0.7$ & $4.7 \pm 1.0$ & $1.8 \pm 0.7$ \\
0162160501 & $4.5 \pm 0.7$ & $1.8 \pm 0.4$ & $4.5 \pm 0.7$ & $1.8 \pm 0.4$ \\
\hline
\end{tabular}

Notes.

${ }^{a}$ Data are first modified by subtracting half of the minimum observed intensity for each direction and each line and then scaled for the observed variation of the HDF-N flux and finally normalized by the model HDF-N SWCX flux.

${ }^{\mathrm{b}}$ Scaled only by the observed variation of the HDF-N flux.

${ }^{c}$ Scaled by the observed variation of the HDF-N flux and then scaled again by the model variation of the HDF-N SWCX flux.

\subsection{Solar Wind Data}

The ACE and WIND satellites at the L1 point provide density and velocity (among other quantities) measurements of various species in the SW. Since SWCX emission is proportional to the flux of the SW species producing the emission, the $A C E$ and WIND data can be used to gain insight into the variation of the observed flux. Figure 5 shows the SW proton flux and the $\mathrm{O}^{7+} / \mathrm{O}^{6+}$ density ratio for the period of this program (the observation intervals are shown by the vertical lines).

Both the SW proton flux and the oxygen density ratio show significant but not unusual variations over the interval. It is unfortunate that the data which are the most relevant, the actual $\mathrm{O}^{8+}$ and $\mathrm{O}^{7+}$ fluxes are not readily available from the $A C E$ instruments and the two displayed parameters must act as surrogates for at least a qualitative understanding of the circumstances. The data normalization that we described in the previous section is associated with the relative values of the proton flux and $\mathrm{O}^{7+} / \mathrm{O}^{6+}$ density ratio between the three observations. The daily average of the SW flux the day before each observation is SEP- $[1,2,3]=[2.6,6.0,1.6] \times 10^{8} \mathrm{~cm}^{-2} \mathrm{~s}^{-1}$ and the equivalent $\mathrm{O}^{7+} / \mathrm{O}^{6+}$ density ratio is $[0.06,0.084,0.025]$. Because of the greater path lengths from the Sun to points along the perpendicular to the Earth-Sun line (i.e., the geometry of the focusing-cone observations), the relevant SW conditions are earlier in time by $\sim 1$ day than those measured at Earth's L1 position, i.e., the location of the ACE spacecraft. Both quantities are lower in the day leading up to the third observation group than the similar periods before the first and second

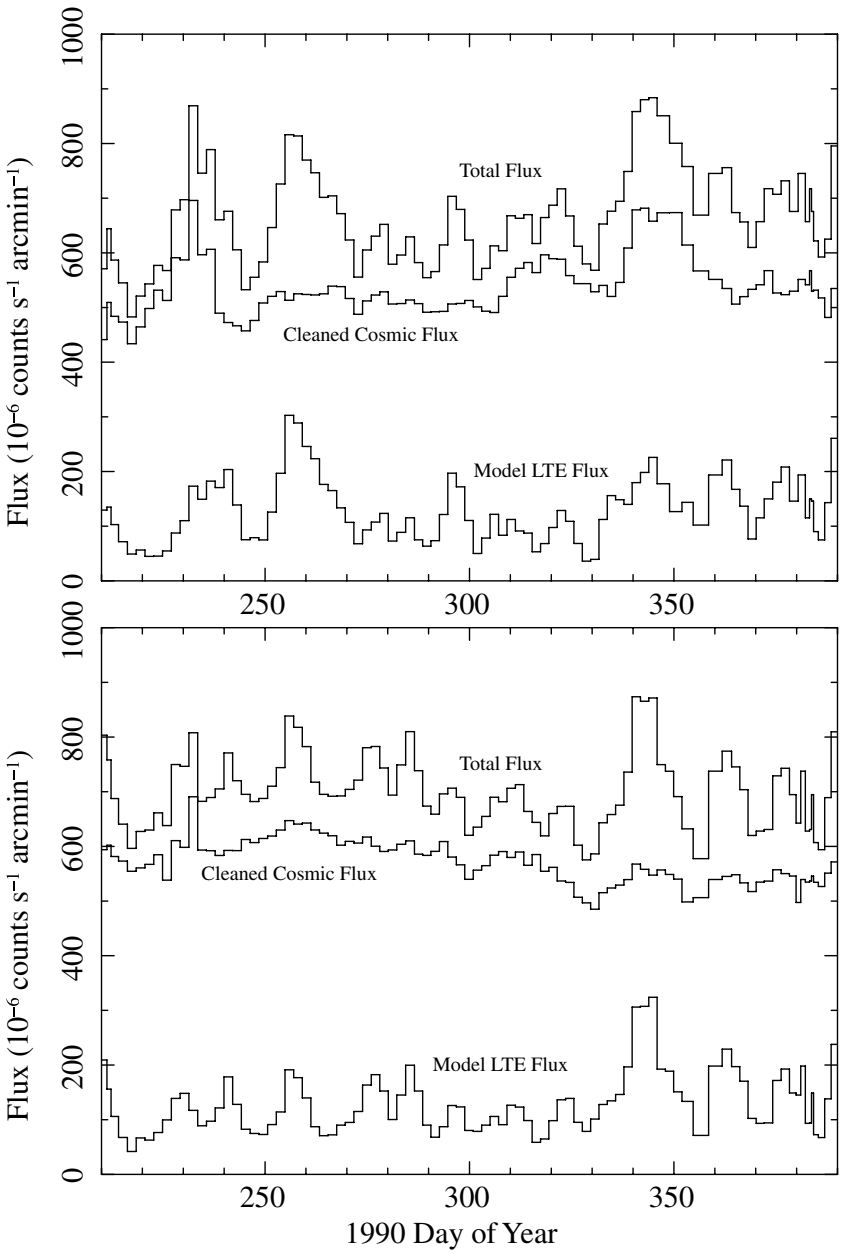

Figure 6. Upper panel: 2 day average RASS $\frac{1}{4} \mathrm{keV}$ surface brightnesses for the intervals of the scans within $2^{\circ}$ of the SEP. In each panel, the top curve is the total measured surface brightness, the lowest curve is the LTE surface brightness (Snowden et al. 1997), and the middle curve is the cosmic (non-LTE) surface brightness. Lower panel: same as the upper panel except the data are from the NEP. Note that day 366.0 of 1990 is day 1.0 of 1991

observation groups, with the highest values leading up to the second observation group. This provides an observational basis for our scaling of the fitted $\mathrm{O}$ VII and $\mathrm{O}$ VIII fluxes with respect to the HDF-N observations.

\subsection{ROSAT All-Sky Survey Ecliptic Pole Data}

In addition to the XMM-Newton observations of the $\mathrm{He}$ focusing cone we also examined the RASS data from the ecliptic poles. Figure 6 shows the total $\frac{1}{4} \mathrm{keV}$ count rate, the LongTerm Enhancement ${ }^{6}$ (LTE; Snowden et al. 1997) and LTEsubtracted RASS $\frac{1}{4} \mathrm{keV}$ surface brightnesses as a function of day of year for the SEP and North Ecliptic Pole (NEP). The data are from periods where the satellite pointing directions were within $2^{\circ}$ of the poles and are 2 day averages. The removal of the LTE enhancements in essence applied a low-pass filter to the data with a time constant on the order of a day. While some of the temporal variation in the "clean" data is possibly due to variation in the cosmic background of distant origin (i.e., beyond the heliosphere), most is due to variations in the SWCX emission and the variable success in removing it from the RASS.

\footnotetext{
6 Long-term Enhancements are the name given to the obvious SWCX enhancements which affected the RASS data. At the time (early 1990s) the origin of this temporally varying "background" enhancement was unknown.
} 
Indeed, had the empirical removal of the SWCX emission been perfectly successful, the cleaned data of the SEP and NEP fields (middle curves in both panels of Figure 6) should show no significant variation. Of note, however, are the enhancements in both the SEP and NEP data near day 345 in the total observed flux and a corresponding enhancement in the cleaned flux for the SEP. These are the strongest enhancements in terms of the integrated magnitude with the southern enhancement being somewhat stronger. Day 345 corresponds to the time period when Earth, and therefore ROSAT, were over the central direction of the He cone. The SW data in the early 1990s, the time period of the RASS observations (the last half of 1990), are too sparse (see Figure 10) to allow a significant quantitative analysis of the data. However, qualitatively the RASS data are in agreement with a model for enhanced SWCX emission during the period when ROSAT observed through the helium focusing cone.

\section{SWCX MODEL DESCRIPTION}

The heliospheric SWCX model we use for our simulations is extensively described in Koutroumpa et al. (2006, 2007). This model is a self-consistent calculation of the SWCX $\mathrm{X}$-ray line emission for any LOS through the heliosphere and for any observation date, based on three-dimensional grids of the IS neutral species ( $\mathrm{H}$ and $\mathrm{He}$ ) distributions in the heliosphere modulated by solar activity conditions (gravity, radiation pressure, and ionization processes). Highly charged heavy SW ions are propagated radially through these grids and the charge-transfer collision rates are calculated for each of the ion species, including the evolution of their density due to charge-transfer with the IS atoms. With this process, we establish three-dimensional emissivity grids for each SW ion species, using photon emission yields computed by Kharchenko \& Dalgarno (2000) for each spectral line following charge exchange with the corresponding neutral species $(\mathrm{H}$ and $\mathrm{He}$ individually). Finally, the X-ray line emission is integrated along any LOS and observation geometry (for each observation date) in order to build the complete spectrum of SWCX emission in the given direction. For comparison to present X-ray observations we use the $\mathrm{O}$ VII triplet at $0.57 \mathrm{keV}$ and the $\mathrm{O}$ VIII line at $0.65 \mathrm{keV}$, as they are the strongest spectral features and provide the best signal-to-noise ratio for the observations.

In Koutroumpa et al. (2007) a dynamic variant of the model is used to simulate quasi real-time SW variations, but in the present analysis the SEP data are normalized with the HDF-N observations (see Section 2.2) in order to account for the actual SW variations. Therefore, we only use the stationary heliospheric SWCX simulations. In Section 4.1, we discuss the success of the data normalization and how the stationary model can fit the normalized data correctly. Nevertheless, the model does not account for SWCX emission from the geocorona and is unable to predict the near-Earth environment reactions to the SW stimulus.

We have conducted three simulations for each of the $X M M$ Newton observations that we name Models A, B, and C. For all simulations the initial parameters of the IS neutral $\mathrm{H}$ and He flows are identical. The parameters specifying the IS neutral hydrogen are: $n_{H}($ at $100 \mathrm{AU})=0.1 \mathrm{~cm}^{-3}, T=13,000 \mathrm{~K}$, $V_{o}=21 \mathrm{~km} \mathrm{~s}^{-1}, \lambda_{\mathrm{UW}}=252.3, \beta_{\mathrm{UW}}=8.5$ (Lallement et al. $2005)$, where $\lambda_{\mathrm{UW}}$ and $\beta_{\mathrm{UW}}$ are the helioecliptic longitude and latitude, respectively, for the upwind direction of the incoming neutral $\mathrm{H}$ flow. The IS helium distribution has the following initial parameters: $n_{\mathrm{He}}($ at $100 \mathrm{AU})=0.015 \mathrm{~cm}^{-3}, T=6300 \mathrm{~K}$,
$V_{o}=26.2 \mathrm{~km} \mathrm{~s}^{-1}, \lambda_{\mathrm{UW}}=254.7, \beta_{\mathrm{UW}}=5.3$ (Witte 2004; Vallerga et al. 2004; Gloeckler et al. 2004). With those parameters, the He focusing cone is expected to be centered around $\left(\lambda_{\mathrm{DW}}, \beta_{\mathrm{DW}}\right)=(74.7,-5.3)$. Model A assumes average solar minimum conditions (e.g., years 1996-1997 or 2007-2008), Model B assumes solar conditions typical for late 2003, and Model C assumes average solar maximum conditions (e.g., years 1990 and 2000-2001). The basic differences in the three simulations are the neutral density distributions as they are shaped by the solar activity and ionization processes and the SW heavy-ion distribution.

The $\mathrm{H}$ density reflects the action of gravity, radiation pressure, and losses due to CX collisions with $\mathrm{SW}$ protons and solar EUV ionization. The ratio, $\mu$, of radiation pressure to gravity for neutral $\mathrm{H}$ varies from 0.9 at solar minimum to 1.5 at solar maximum (Woods et al. 2000). The main source of ionization for $\mathrm{H}$ atoms is $\mathrm{CX}$ with $\mathrm{SW}$ protons. Ionization rates are derived as a function of heliographic latitude from the Solar and Heliospheric Observatory (SOHO)/SWAN Ly- $\alpha$ preliminary data analysis (Quémerais et al. 2006). The source of the latitudinal variation of the ionization rates is in fact the latitudinal variation in the SW. For solar minimum the ionization rate has a $40 \%$ relative decrease from the solar equator to the solar poles, with an equatorial value of $6.7 \times 10^{-7} \mathrm{~s}^{-1}$, while in solar maximum the relative decrease from the equator to the poles is only around $15 \%$, with an equatorial value of $8.4 \times 10^{-7} \mathrm{~s}^{-1}$. In late 2003 , with $\mu \sim 1.2$, the equatorial ionization rate is $7.5 \times 10^{-7} \mathrm{~s}^{-1}$ and the equator to pole relative decrease is $30 \%$ in the $\mathrm{H}$ ionization rates.

For $\mathrm{He}$ atoms, the radiation pressure to gravity ratio is effectively zero and the atoms are gravitationally focused downwind (the helium focusing cone). The main cause of ionization is solar EUV radiation and electron impact. Recent work has shown strong evidence for a latitudinally anisotropic distribution of the $\mathrm{He} 30.4 \mathrm{~nm}$ solar irradiance and thus of the He photoionization rate (Witte 2004; Auchère et al. 2005) and it has also been shown that the electron impact ionization rate is also dependent on heliographic latitude (McMullin et al. 2004). In Koutroumpa et al. (2007), we tested preliminary grids of anisotropic He distributions in our calculation of heliospheric X-rays induced in SWCX collisions. The present study, which examines emission from the He focusing cone, attempts to constrain the He ionization rates and their latitudinal dependence.

For maximum solar activity, the photoionization rate is $1.4 \times 10^{-7} \mathrm{~s}^{-1}$ at the solar equator, while during solar minimum the photoionization rate at the solar equator is $0.7 \times 10^{-7} \mathrm{~s}^{-1}$. The photoionization rates present a $50 \%$ decrease toward the solar poles. In 2003 , the He photoionization rate was $1 \times$ $10^{-7} \mathrm{~s}^{-1}$ at the solar equator, again with a $50 \%$ decrease at the solar poles.

The radial dependence of electron impact ionization is taken from Rucinski \& Fahr (1989) which is appropriate for solar minimum conditions but requires a threefold increase for solar maximum (Lallement et al. 2004). For this analysis we include a heliographic latitude dependence correction factor, derived from McMullin et al. (2004), that we scale from minimum to maximum. The Lallement et al. (2004) scaling factor in combination with the latitudinal anisotropy factor applied to the Rucinski \& Fahr (1989) electron impact model is given in Table 4. An additional simulation (labeled B1) was also performed which was identical to Model B except without electron impact ionization. 
Table 4

Heliographic Latitude-dependent Coefficients

\begin{tabular}{lccc}
\hline \hline $\begin{array}{l}\text { Heliographic } \\
\text { Latitude }\left(^{\circ}\right)\end{array}$ & $\begin{array}{c}\text { Model A } \\
\text { Minimum (2008) }\end{array}$ & $\begin{array}{c}\text { Model B } \\
\text { Medium (2003-2004) }\end{array}$ & $\begin{array}{c}\text { Model C } \\
\text { Maximum (2001) }\end{array}$ \\
\hline 80 & 0.6 & 0.6 & 2.4 \\
60 & 0.65 & 0.65 & 2.4 \\
40 & 0.8 & 0.8 & 2.7 \\
20 & 0.9 & 0.9 & 2.85 \\
0 & 1.0 & 1.0 & 3.0 \\
-20 & 0.9 & 0.9 & 2.85 \\
-40 & 0.8 & 0.8 & 2.7 \\
-60 & 0.65 & 0.65 & 2.4 \\
-80 & 0.6 & 0.6 & 2.4 \\
\hline
\end{tabular}

Note. The coefficients are applied to the Rucinski \& Fahr (1989) electron impact ionization rate for $\mathrm{He}$ atoms and they include the threefold increase from solar minimum to solar maximum found by Lallement et al. (2004), as well as the latitude dependence factor derived from McMullin et al. (2004).

The latitude dependence of the SW also affects the highly charged heavy-ion distribution, where abundances depend on the SW type. During minimum solar activity, the SW is considered to be highly anisotropic, with a narrow equatorial zone (within $\pm 20^{\circ}$ of the solar equatorial plane) of slow SW with an average speed of $\sim 400 \mathrm{~km} \mathrm{~s}^{-1}$ and the fast SW emitted from the polar coronal holes at a speed of $\sim 700 \mathrm{~km} \mathrm{~s}^{-1}$. The slow SW has a proton density of $\sim 6.5 \mathrm{~cm}^{-3}$ at $1 \mathrm{AU}$, while the fast flow is less dense at $\sim 3.2 \mathrm{~cm}^{-3}$ at $1 \mathrm{AU}$. At solar maximum, the SW spatial distribution is considered to be a complex mix of slow and fast wind states that is in general approximated with an average slow wind flux. The ionic composition of the two flows can be very different with the average oxygen content varying from $[\mathrm{O} / \mathrm{H}]=1 / 1780$ in the slow wind and $[\mathrm{O} / \mathrm{H}]=1 / 1550$ in the fast flow. The charge state distributions change as well, with the higher charge states strongly depleted (or even completely absent, as for example $\mathrm{O}^{8+}$ ) in the fast SW. For our model, we adopt the oxygen relative abundances published in Schwadron \& Cravens $(2000):\left(\mathrm{O}^{7+}, \mathrm{O}^{8+}\right)=(0.2,0.07)$ for the slow wind and $\left(\mathrm{O}^{7+}, \mathrm{O}^{8+}\right)=(0.07,0.0)$ for the fast wind, based on data from the Ulysses SWICS instrument (Schwadron et al., 2000, unpublished document).

The main difference in the SW heavy-ion distribution in our three simulations is the spatial (latitudinal) distribution of the slow and fast SW flows. For Model A (minimum SW) the slow SW is expanding in interplanetary space through a $\pm 20^{\circ}$ equatorial zone on the solar surface, while the fast SW flow occupies the rest of the space. During solar maximum (Model C), we have considered that only an isotropic slow SW flow is present in the interplanetary space. For the late 2003 period (Model B) we assume that there is no fast wind flow in interplanetary space, in order to estimate average upper limits (we also assume that there is no coronal mass ejection (CME) or abnormal abundance distributions at the time of the XMMNewton observations) for the resulting SWCX X-ray emission. Indeed, as demonstrated in Koutroumpa et al. (2006, 2007), for high ecliptic latitude LOS, as is the case for the XMM-Newton He cone observations $\left(\beta \sim-90^{\circ}\right)$, the oxygen line intensity decreases from solar maximum to solar minimum conditions as the LOS crosses larger fast wind regions where the parent ions are strongly depleted. Thus, finally, the difference between Models B and C lies only in the different ionization rates for the neutral species, as they were defined earlier in this section.

The model results for the SEP observations are listed in Table 5. The highest on-cone SWCX excess is found for the minimum solar conditions Model A ( $0.7 \mathrm{LU}$ and $0.3 \mathrm{LU}$ for $\mathrm{O}$ VII and $\mathrm{O}$ VIII, respectively), where the neutral density is expected to be the highest. However, in the most realistic case for late 2003 (Model B/B1) the total on-cone excess predicted by the simulations is $0.5 \mathrm{LU}$ for $\mathrm{O}$ VII and $0.2 \mathrm{LU}$ for $\mathrm{O}$ VIII. In the same table, we also list the HDF-N simulation results for Model B.

\section{DISCUSSION}

\subsection{Comparison to XMM-Newton data}

In Figure 7, we compare the data and model results for the oxygen line intensities for the three XMM-Newton SEP observations. All the simulations predict the maximum line emission on the second observation date, when the Earth is located at $72^{\circ}$ of ecliptic longitude. The nominal position of the He cone is at $\sim 75^{\circ}$, only $3^{\circ}$ from the second XMM-Newton observation date. We also include the Model B prediction for the HDF-N pointings during the observational campaign. If we take into account the HDF-N variation predicted in the SWCX model and scale the XMM-Newton data we find that the data yield the same trend as the model predictions, both for the first scaling of the data and for where half of the minimum observed emission is assumed to be of cosmic origin. If the measured data are assumed to be dominated by the heliospheric component, the model can only yield roughly half of the observed absolute intensity levels. Also, the on-cone SWCX excess with respect to the off-cone directions predicted in the four simulations is much

Table 5

Model Oxygen Line Intensities in LU

\begin{tabular}{|c|c|c|c|c|c|c|c|c|c|}
\hline \multirow[t]{2}{*}{ ObsId } & \multirow[t]{2}{*}{ Target } & \multicolumn{2}{|c|}{ Model $A^{\mathrm{a}}$} & \multicolumn{2}{|c|}{ Model B ${ }^{b}$} & \multicolumn{2}{|c|}{ Model B1 ${ }^{\mathrm{c}}$} & \multicolumn{2}{|c|}{ Model $C^{d}$} \\
\hline & & O VII & O VIII & O VII & O VIII & O VII & O VIII & O VII & O VIII \\
\hline 0162160101 & SEP & 1.7 & 0.4 & 3.3 & 1.3 & 3.4 & 1.3 & 2.5 & 1.0 \\
\hline 0162160201 & HDF-N & $\ldots$ & $\ldots$ & 3.2 & 1.3 & $\ldots$ & $\ldots$ & $\ldots$ & $\ldots$ \\
\hline 0162160301 & SEP & 2.4 & 0.7 & 3.8 & 1.5 & 4.0 & 1.5 & 3.0 & 1.2 \\
\hline 0162160401 & HDF-N & $\ldots$ & $\ldots$ & 3.2 & 1.3 & $\ldots$ & $\ldots$ & $\ldots$ & $\ldots$ \\
\hline 0162160501 & SEP & 1.9 & 0.5 & 3.6 & 1.4 & 3.8 & 1.5 & 2.8 & 1.1 \\
\hline 0162160601 & HDF-N & $\ldots$ & $\ldots$ & 2.9 & 1.1 & $\ldots$ & $\ldots$ & $\ldots$ & $\ldots$ \\
\hline
\end{tabular}

Notes.

${ }^{a}$ Minimum SW conditions (e.g., 2008).

${ }^{b}$ Medium SW conditions (e.g., 2003-2004).

${ }^{c}$ Medium SW conditions (e.g., 2003-2004) with no electron impact ionizations.

d Maximum SW conditions (e.g., 2001). 

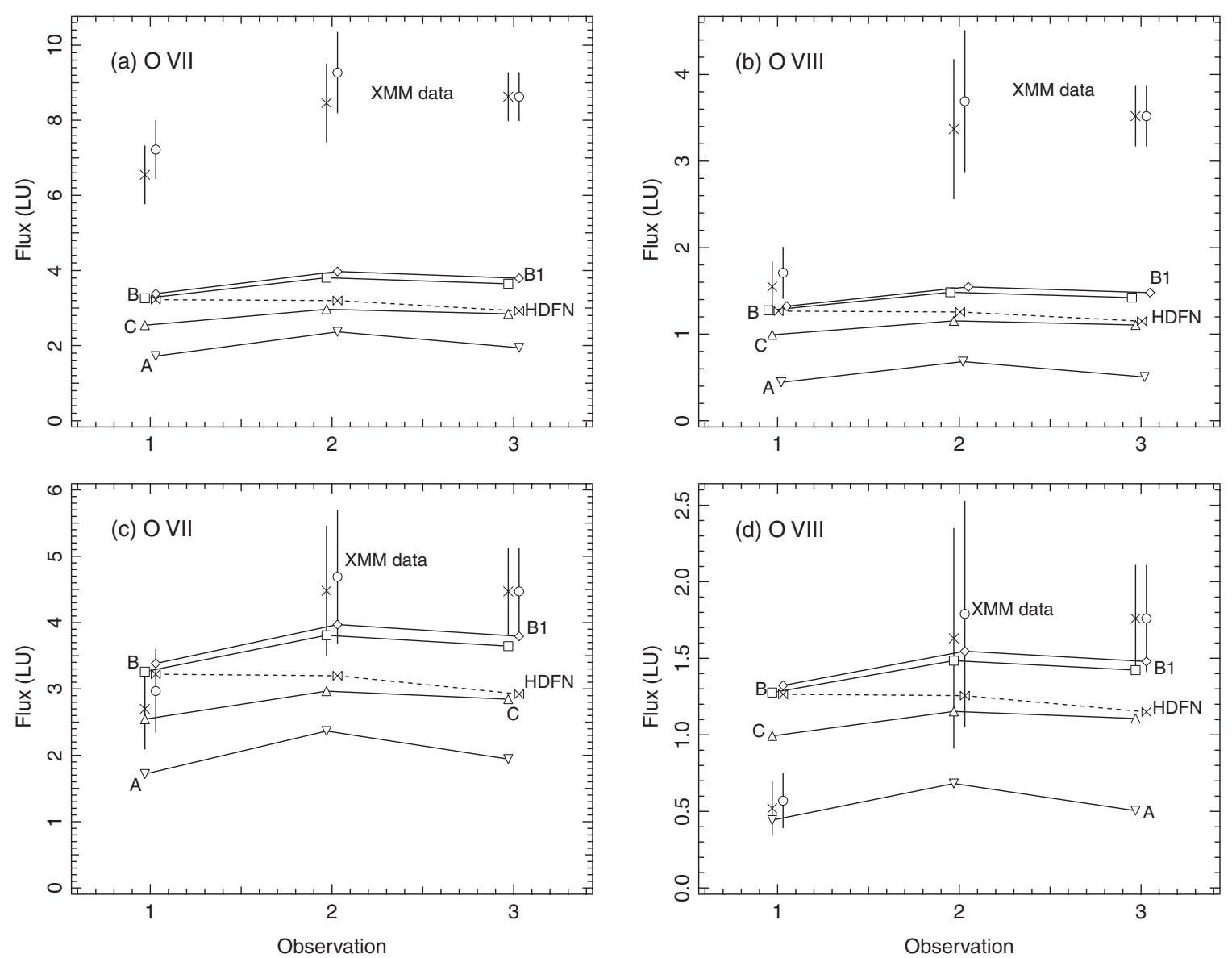

Figure 7. (a) Model and data line intensities for the O vII triplet in the direction of the SEP. The data are from Table 2. Data points with an X symbol show the results when the HDF-N SWCX flux is assumed to be constant. The points with a circle symbol include a further scaling to account for the model variation in the direction of the HDF-N (double-triangle symbol). The model points are from Table 5. Inverse triangle symbols are for Model A, square symbols are for Model B, diamond symbols for Model B1, and triangle symbols are for Model C. (b) Same as (a) but for the O vIII line. (c) and (d) are the same as (a) and (b), respectively, except for the assumption that half of minimum observed flux in both directions has an origin outside of the heliosphere.

lower than what is found in the data. In addition, we need to note again that the model calculates only the heliospheric SWCX component, and it is possible to have some residual variations due to SWCX emission from the near-Earth environment. Moreover, the data statistics are relatively poor, and the oncone to off-cone difference is marginal, due to the large error bars.

That the model absolute intensities underpredict the data (when no contribution from the cosmic background is assumed) is not surprising since the observed field is not shadowed from distant emission, such as from the galactic halo, as we explained previously in Section 2.2. If we subtract the Model $(M)$ intensities from the Data $(D)$, we obtain the residual cosmic background intensity. Assuming that the SWCX emission in the $\mathrm{He}$ cone is the only source of variation in the measured data, and ignoring the small differences eventually due to the look direction, the residual Data $(D)-\operatorname{Model}(M)$ cosmic background should remain constant over the time. In our initial analysis, this is not the case either for $\mathrm{O}$ VII or for O VIII. However, if we assume that the expected constant background (here after named $R$ ), for each simulation, is equal to the average $\langle D-M\rangle$ (Data-Model) difference between the three observation dates $(R=\langle D-M\rangle)$, then the model intensities for each simulation need to be multiplied by a correction factor $\alpha$ (as shown in the upper panels of Figure 8), such that

$$
D_{i}=R+\alpha \cdot M_{i}
$$

for each SEP observation $i$. Since, neither the average $R$ value representing the residual cosmic background, nor the corrected SWCX emission $(\alpha \cdot M)$ is allowed to have negative values, we assume that the average residual cosmic background $R$ cannot exceed the lower normalized value detected with XMM-Newton for each of the oxygen lines $(7.2$ and $1.7 \mathrm{LU}$, respectively, for $\mathrm{O}$ VII and $\mathrm{O}$ VIII). A correction factor $\alpha$ less than 1 (respectively more than 1) means that the model SWCX emission is overestimated (underestimated) and needs to be lowered (increased) in order to achieve a constant residual background $R$. The closer the correction factor $\alpha$ is to unity, the least correction is needed for the model to reproduce the data.

In our initial analysis, the correction required for the simulation results to match the measured data is dominated by the large differences between the data and model absolute intensities, and the correction factors for all four simulations are very similar. Moreover, the correction factor error bars (calculated based on error propagation from the XMM-Newton data standard variations) overlap for all simulations, revealing that there is little difference between our four models.

In the more realistic case, where we assumed that half of the observed flux is of cosmic origin, the second data set (that we name $D^{\prime}$ ) may be directly compared with the SWCX simulation results $M$, and the correction required for the model to match the data set may be simplified as $D_{i}^{\prime}=\alpha^{\prime} \cdot M_{i}$. The $\alpha^{\prime}$ correction 

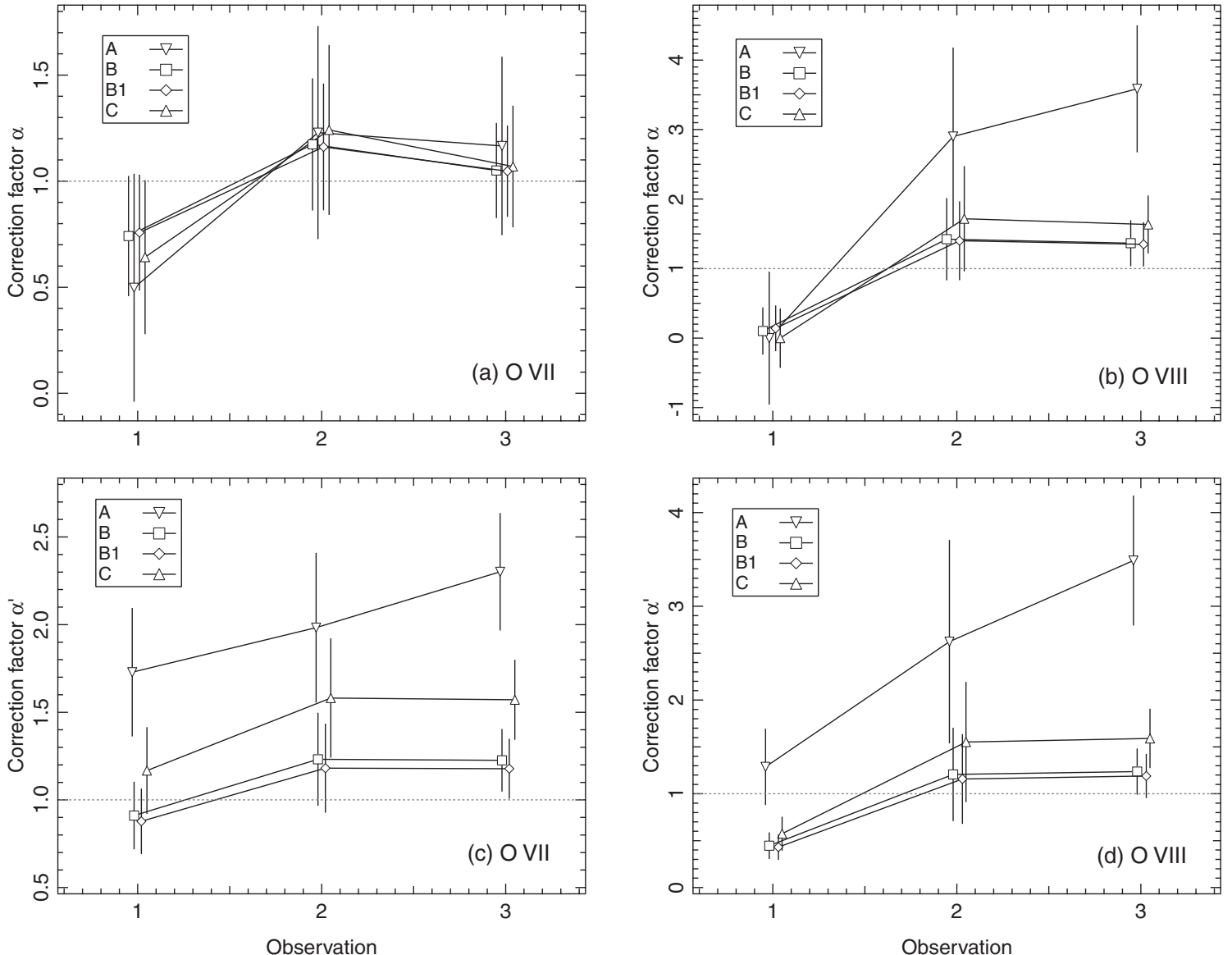

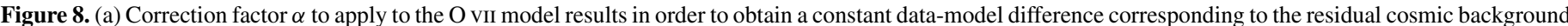

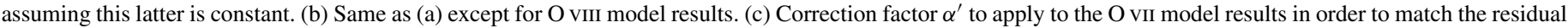

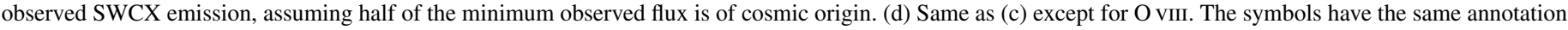
as in Figure 7.

factors are presented in the lower panels of Figure 8. In this case, the correction factors have a broader distribution and the error bars overlap to a lesser extent, allowing a better determination of the best-fit simulation.

From both factors $\alpha$ and $\alpha^{\prime}$ in Figure 8, we find that the least correction (best fit, factors closer to unity) is found for the simulations B/B1 (solar activity adjusted for late 2003) which is in good agreement with the data, especially for the $\mathrm{O}$ VII line. The model requiring the largest correction is the one accounting for solar minimum activity period (Model A), which also assumes a highly anisotropic distribution for the $\mathrm{SW}$ oxygen ions. We also see that results for the O VII triplet need smaller corrections than the $\mathrm{O}$ viII results. The difficulty to fit the $\mathrm{O}$ VIII data with the model most likely arises from the poor statistics of the XMM-Newton $\mathrm{O}$ viII data, and the large uncertainties (or complete lack) of SW data for $\mathrm{O}^{8+}$ that serve as input in the SWCX simulations.

Our results suggest that toward high ecliptic latitudes in late 2003, there was little fast wind, and a slow wind flux with high oxygen content is required to reproduce the SWCX X-ray increase in the He cone. In order to investigate this assumption, we calculate the correction factors needed by Model B to be scaled for different latitudinal extents of the slow SW, and we plot the results for line O VII in Figure 9. Starting at an SW latitudinal extent of $\pm 30^{\circ}$ the correction factor progressively improves to its best fit for the three exposures, obtained for an isotropic slow wind (latitudinal extent of $\pm 90^{\circ}$ around

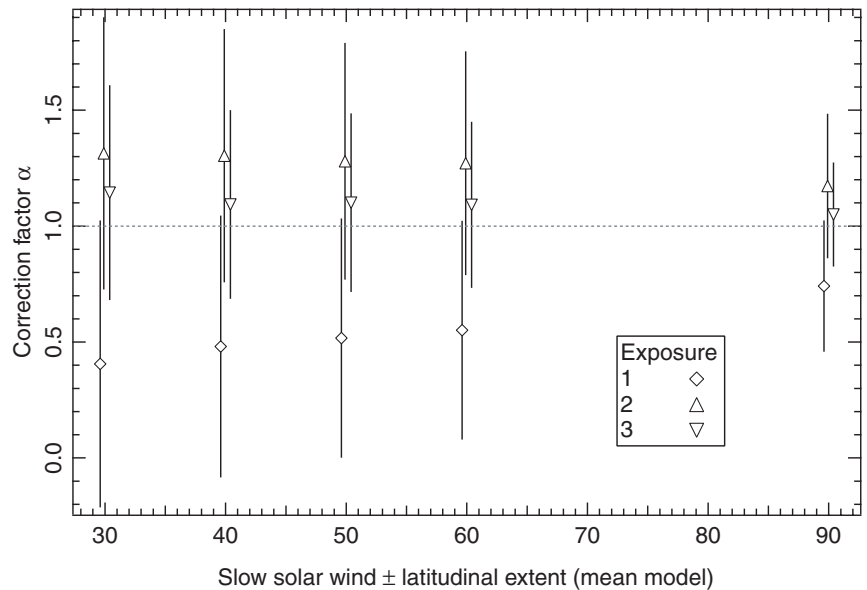

Figure 9. Correction factors to apply to Model B results for different latitudinal slow SW extent.

the solar equator). However, any correction factor variation remains within error bars, and could eventually be considered nonsignificant.

\subsection{Comparison to ROSAT $\frac{1}{4}$ keV Data}

In Figure 10, we compare the ROSAT $\frac{1}{4} \mathrm{keV}$ surface brightness as a function of the observation date, with the equivalent SWCX model $\frac{1}{4} \mathrm{keV}$ surface brightness. The SWCX simulated spectra 

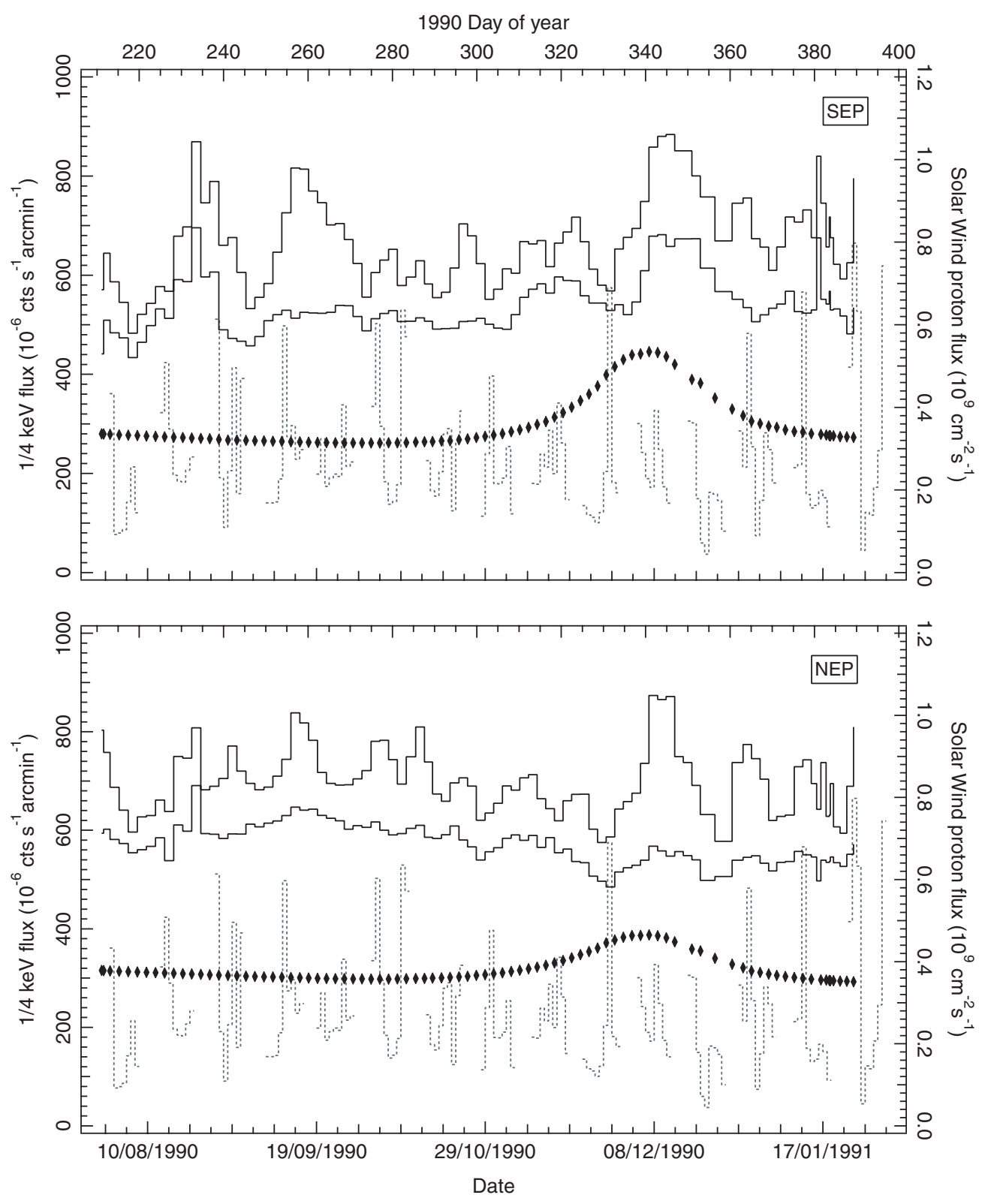

Figure 10. Upper panel: model and data $\frac{1}{4} \mathrm{keV}$ surface brightnesses for the SEP. The plain curves are the total (upper) and LTE-corrected (lower) RASS $\frac{1}{4}$ keV fluxes. The diamonds are the SWCX model $\frac{1}{4} \mathrm{keV}$ fluxes for the same direction. On the right axis (dotted curve), we present daily averages of the SW proton flux during the same period. Lower panel: same as the upper panel except the data and model results are for the NEP. Day 366.0 of 1990 is day 1 of 1991.

toward the NEP and SEP, for each ROSAT observation date, were convolved with the ROSAT $\frac{1}{4} \mathrm{keV}$ band response in order to obtain the surface brightness due to SWCX emission for these dates and LOS. The first thing to note is that the model shows an expected enhancement for both the SEP and NEP directions. This is not unreasonable as the He cone while centered along a line at -5.5 ecliptic latitude has an angular extent of $\sim \pm 25^{\circ}$. Comparing the model to the data shows a qualitative agreement with enhancements in the total observed flux (upper curve), also in both directions, and indeed they are the strongest enhancements in terms of their magnitude and duration. However, considering both the cleaned and uncleaned data, the SEP and NEP peak locations in the data are slightly offset at higher longitudes ( $\sim 5$ days) with respect to the model. This is a marginal result but is consistent with additional SWCX emission from the secondary He cone. However, this offset could also be the effect of a poorly timed enhancement of the SW, as the He cone reacts fairly quickly to SW enhancements (see Figures 2 and 5 of Cravens et al. 2001), especially toward polar look directions. Indeed, the cleaned data (lower curve) indicate that the NEP enhancement was more effectively removed than that for the $\mathrm{SEP}^{7}$ in the empirical cleaning process.

\section{CONCLUSIONS}

We specifically designed a series of XMM-Newton observations in order to observe SWCX emission from the helium focusing cone in the heliosphere. Three observations of the SEP, coupled with three monitoring observations of the HDF-N, were

\footnotetext{
7 Data from the southern hemisphere were more sparse than from the north as data losses from passage through the South Atlantic Anomaly preferentially affected the south, and this in turn affected the empirical cleaning process.
} 
performed. The HDF-N observations were used to normalize the SEP data, in order to attempt to eliminate short-term variation effects of the SW. After normalizing the SEP data using the HDF-N data, we observe an SWCX heliospheric excess (with respect to the off-cone directions) of $2.1 \pm 1.3 \mathrm{LU}$ for $\mathrm{O}$ VII and $2.0 \pm 0.9 \mathrm{LU}$ for $\mathrm{O}$ VIII when we assume that the lines are dominated by SWCX emission from the heliosphere. If we assume that half of the observed emission is of cosmic origin, we find an SWCX excess of $1.7 \pm 1.2 \mathrm{LU}$ and $1.2 \pm 0.7 \mathrm{LU}$ for $\mathrm{O}$ VII and $\mathrm{O}$ VIII lines, respectively. The maximum emission is observed on the second XMM-Newton observation, when the observatory (Earth) was located at $72^{\circ}$ of ecliptic longitude. This location is consistent with the nominal central position of the He cone $\left(\sim 74^{\circ}\right)$. We find no clear evidence of the secondary He cone reported in Collier et al. (2004), but the data are not inconsistent with such a component either.

We compared the data results to a self-consistent model of the SWCX X-ray emission in the heliosphere, taking into account both $\mathrm{H}$ and $\mathrm{He}$ neutral components in the interplanetary space and also accounting for solar activity variations. The model predicted an equivalent trend between the three observations, with the maximum emission occurring at $72^{\circ}$ of ecliptic longitude. The average absolute intensity predicted by the model is roughly half of the measured total intensity, since the field of view does not include any shadowing structures (i.e., dense molecular clouds) that would absorb the more distant halo emission. However, the on-cone excess predicted by the model (assuming solar conditions close to the late 2003 period, when the $X M M$-Newton observations were performed) is much smaller with respect to the data on-cone excess, only $0.5 \mathrm{LU}$ and $0.2 \mathrm{LU}$ for $\mathrm{O}$ VII and $\mathrm{O}$ VIII respectively.

This discrepancy between the model results and the data may be attributed to residual short-scale SW variations from geocorona SWCX emission that the model does not account for. The $A C E$ and Wind data in the ecliptic plane at $1 \mathrm{AU}$ displayed significant variations during this period, but within reasonable limits. However, considering the high ecliptic latitude observation geometry, both for the SEP and the HDF-N pointings, we cannot assert that the SW conditions were indeed the same as those measured in the ecliptic, or that both the SEP and HDF-N observations were influenced by SW of the same characteristics. In order to quantify the model to data discrepancy, we produced a series of scaling factors needed by the different simulations to match the data. The correction factors for the simulations accounting for the late 2003 period of solar activity show a reasonable agreement of the model with the data.

We also compared a series of RASS data toward the SEP and NEP with the SWCX model results in the $\frac{1}{4} \mathrm{keV}$ band. The data (both NEP and SEP) exhibit a clear enhancement near the nominal He cone position predicted in the model. The SEP data also show a shoulder toward higher longitude which may be consistent with the presence of the secondary He cone. However, it must be noted that the shoulder could also be due to a serendipitous enhancement of the SW.

More X-ray observations of the He cone, especially during different solar activity periods, are needed to further constrain the SWCX emission from the He cone and the heliosphere in general. Hopefully, the study will be reinforced by spectral diagnostics with the help of future X-ray missions including calorimeter instruments.

This study is based on observations obtained with $X M M$ Newton, an ESA Science Mission with instruments and contri- butions directly funded by ESA Member States and NASA. We are grateful to the ESA XMM-Newton SOC for supporting and planning the special calibration observations used in this paper.

This research was supported by an appointment to the NASA Postdoctoral Program at the Goddard Space Flight Center, administered by Oak Ridge Associated Universities through a contract with NASA.

The authors thank M. Bzowski for constructive and very useful discussions on the neutral gas distributions. We are also grateful to the anonymous referee for his/her pertinent questions and useful remarks that considerably improved the manuscript.

\section{REFERENCES}

Auchère, F., Cook, J. W., Newmark, J. S., McMullin, D. R., von Steiger, R., \& Witte, M. 2005, ApJ, 625, 1036

Bzowski, M., Fahr, H. J., \& Rucinski, D. 1996, Icarus, 124, 209

Collier, M. R., et al. 2004, Adv. Space Res., 34, 166

Cox, D. P. 1998, in IAU Coll. 166, The Local Bubble and Beyond, Lecture Notes in Physics, Vol. 506, ed. D. Breitschwerdt, M. J. Freyberg, \& J. Truemper (Berlin: Springer-Verlag), 121

Cravens, T. E. 2000, ApJ, 532, L153

Cravens, T. E., Robertson, I. P., \& Snowden, S. L. 2001, J. Geophys. Res., 106, 24883

Frisch, P. C. 2000, J. Geophys. Res., 105, 10279

Gloeckler, G., et al. 2004, A\&A, 426, 845

Gruntman, M. A. 1994, J. Geophys. Res., 99, 19213

Holmström, M., Collier, M. R., Barabash, S., Brinkfeldt, K., Moore, T. E., \& Simpson, D. 2008, Adv. Space Res., 41, 343

Kharchenko, V. 2005, in AIP Conf. Proc. 774, X-ray Diagnostics of Astrophysical Plasmas: Theory, Experiment, and Observation, ed. R. Smith (New York: AIP), 271

Kharchenko, V., \& Dalgarno, A. 2000, J. Geophys. Res., 105, 18351

Koutroumpa, D., Acero, F., Lallement, R., Ballet, J., \& Kharchenko, V. 2007, A\&A, 475, 901

Koutroumpa, D., Lallement, R., Kharchenko, V., \& Dalgarno, A. 2008, Space Sci. Rev., 87

Koutroumpa, D., Lallement, R., Kharchenko, V., Dalgarno, A., Pepino, R., Izmodenov, V., \& Quémerais, E. 2006, A\&A, 460, 289

Kuntz, K. D., \& Snowden, S. L. 2008, A\&A, 478, 575

Lallement, R. 1999, in AIP Conf. Ser. 471, Proc. of the Ninth International Solar Wind Conference, ed. S. R. Habbal, et al. (Melville, NY: AIP), 205

Lallement, R. 2009, Space Sci. Rev., 143, 427

Lallement, R., Quémerais, E., Bertaux, J. L., Ferron, S., Koutroumpa, D., \& Pellinen, R. 2005, Science, 307, 1447

Lallement, R., Raymond, J. C., Vallerga, J., Lemoine, M., Dalaudier, F., \& Bertaux, J. L. 2004, A\&A, 426, 875

Lisse, C. M., et al. 1996, Science, 274, 205

McMullin, D. R., et al. 2004, A\&A, 426, 885

Moebius, E., Hovestadt, D., Klecker, B., Scholer, M., \& Gloeckler, G. 1985, Nature, 318,426

Ogilvie, K. W., et al. 1995, Space Sci. Rev., 71, 55

Quémerais, E., Bertaux, J. L., Lallement, R., Berthé, M., Kyrölä, E., \& Schmidt, W. 1999, J. Geophys. Res., 104, 12585

Quémerais, E., Lallement, R., Ferron, S., Koutroumpa, D., Bertaux, J. L., Kyrölä, E., \& Schmidt, W. 2006, J. Geophys. Res. (Space Phys.), 111, 9114

Robertson, I. P., \& Cravens, T. E. 2003, Geophys. Res. Lett., 30, 1439

Rucinski, D., \& Fahr, H. J. 1989, A\&A, 224, 290

Schwadron, N. A., \& Cravens, T. E. 2000, ApJ, 544, 558

Smith, R. K., Edgar, R. J., Plucinsky, P. P., Wargelin, B. J., Freeman, P. E., \& Biller, B. A. 2005, ApJ, 623, 225

Snowden, S. L. 2009, Space Sci. Rev., 143, 253

Snowden, S. L., Collier, M. R., Cravens, T., Kuntz, K. D., Lepri, S. T., Robertson, I., \& Tomas, L. 2009, ApJ, 691, 372

Snowden, S. L., Collier, M. R., \& Kuntz, K. D. 2004, ApJ, 610, 1182

Snowden, S. L., Mushotzky, R. F., Kuntz, K. D., \& Davis, D. S. 2008, A\&A, 478,615

Snowden, S. L., et al. 1995, ApJ, 454, 643

Snowden, S. L., et al. 1997, ApJ, 485, 125

Stone, E. C., Frandsen, A. M., Mewaldt, R. A., Christian, E. R., Margolies, D., Ormes, J. F., \& Snow, F. 1998, Space Sci. Rev., 86, 1 
Vallerga, J., Lallement, R., Lemoine, M., Dalaudier, F., \& McMullin, D. 2004, A\&A, 426, 855

Wang, L., Lin, R. P., Larson, D. E., \& Luhmann, J. G. 2008, Nature, 454, 81

Witte, M. 2004, A\&A, 426, 835
Woods, T. N., Tobiska, W. K., Rottman, G. J., \& Worden, J. R. 2000 J. Geophys. Res., 105, 27195

Wurz, P., Collier, M. R., Moore, T. E., Simpson, D., Fuselier, S., \& Lennartson, W. 2004, in AIP Conf. Proc. 719, Physics of the Outer Heliosphere, ed. V. Florinski, N. V. Pogorelov, \& G. P. Zank (Melville, NY: AIP), 195 